\title{
LRS Bianchi Type-I Universe in $F(T)$ Theory of Gravity
}

\author{
M.V. Dawande ${ }^{1}$, K. S. Adhav², S. S. Nerkar ${ }^{3}$ \\ ${ }^{1}$ Bharatiya Mahavidyalaya, Amravati \\ ${ }^{2}$ Professor (Mathematics), School of Mathematical \& Statistical Sciences, Hawassa University, Ethiopia \\ ${ }^{3}$ P.R.Pote (Patil) College of Engineering and Management, Amravati \\ Email: ati_ksadhav@yahoo.co.in, sheetalnerkar03@gmail.com.
}

\begin{abstract}
We have studied the spatially homogeneous and anisotropic Locally Rotationally Symmetric (LRS) Bianchi type-I universe in $F(T)$ theory of gravity. By using a conservation equation, we have discussed some well known $F(T)$ models. It is interesting to observe that these $F(T)$ gravity models represent the different phases (matter, radiation and dark energy eras) of the universe. An attempt has been made to retain Sharif and Rani's [1] forms of the various quantities. Our results are analogous to the results obtained by Sharif and Rani [1].
\end{abstract}

Keywords: $F(T)$ gravity, LRS Bianchi type-I universe, continuity equation.

\section{Introduction}

The observations of Supernova type-Ia experiments [2-6], cosmic microwave background (CMB) anisotropies [7-9], large scale structure [10-12] have proposed that a peculiar component, generally known as the dark energy (DE), with high negative pressure is pushing this present cosmic accelerated expansion. This component occupies $68.3 \%$ of the present universe dominating the other components of the universe. (i.e., dark matter component (26.8\%) and baryonic matter (4.9\%) as observed by PLANCK 2013 [13].

The concept of DE was introduced by Einstein himself when he added the cosmological constant in the field equations. In spite of all observational evidences, explaining expansion of universe has been an open challenge in modern physics [14]. The most suitable of the modified theories is $F(R)$ theory of gravity which is a function of Ricci scalar $R$ in standard Einstein-Hilbert Lagrangian [15-20]. The $F(R)$ theory gives cosmic inflation and depicts nature of dark energy with present cosmic acceleration. Another modified theory developed by Harko et al. [21] is known as $F(R, T)$ which is the generalization of $F(R)$ gravity and depends upon coupling of matter and geometry. Here the Lagrangian includes a function of the scalar curvature $R$ and the trace of energy momentum tensor T. Also, Ferraro and Fiorini $[22,23]$ have developed $F(T)$ theory and solved practical horizontal problem as well as obtained singularity free solutions with positive cosmological constant. This $F(T)$ theory of gravity is the generalization of teleparallel gravity where curvature free Weitzenböck connections are used. This theory reduces to general relativity if $F(T)$ is replaced by a constant $[24,25]$. Although $F(R)$ gravity has given many cosmologically important models [26-28], it is rather challenging as its equations are of fourth order. On the other hand, $F(T)$ produces equations of order two and gives interesting results [29-33].

Our universe is homogeneous and isotropic on large scale. Result obtained by WMAP data [34-36] shows the existence of an anisotropic phase of the universe which approaches isotropy. The anisotropic model has been gaining interest since then. The Bianchi type models are spatially homogeneous and anisotropic. The most basic anisotropic model i.e. the Bianchi Type I (BI) universe has been studied by several researchers to discuss the effect of anisotropy in several contexts. Kumar and Singh [37, 38] have studied the exact solutions for the Bianchi Type I universe in various theories. Sharif and Waheed [39] investigated exact solutions for anisotropic fluid by considering the LRS Bianchi type-I universe which generalizes the flat FRW universe in the modified theory. Work by many researchers on this model is available using different parameters and different theories [40-46].

We have discussed LRS Bianchi type-I models in $F(T)$ gravity. In the second section we have presented some basics of teleparallel gravity and the corresponding field equations for LRS Bianchi Type-I are given in section 3. A detailed construction of $F(T)$ gravity models is given using continuity equation in section 4 . In the last section, we summarize and conclude the results. 


\section{$2 \quad F(T)$ Gravity Formalism}

Let us present $F(T)$ gravity. We introduce the modified teleparallel theory of gravity as well as extension to $F(T)$ theory. The Lagrangian density for teleparallel and $F(T)$ gravity [25] are respectively given by

$$
\begin{aligned}
L_{T} & =-\frac{e}{16 \pi G} T \\
L_{F(T)} & =-\frac{e}{16 \pi G} F(T)
\end{aligned}
$$

where $T$ is the torsion scalar, $F(T)$ is a differentiable function of torsion scalar $T$, $\mathrm{G}$ is the gravitational constant and $e=\sqrt{-g}$.

The torsion has the form

$$
T=S_{\rho}^{\mu \nu} T_{\mu v}^{\rho}
$$

where $S_{\rho}{ }^{\mu v}$ is the antisymmetric tensor; $T^{\rho}{ }_{\mu v}$ is the torsion tensor which are respectively defined by

$$
\begin{gathered}
S_{\rho}^{\mu \nu}=\frac{1}{2}\left(K_{\rho}^{\mu v}+\delta_{\rho}^{\mu} T_{\theta}^{\theta \nu}-\delta_{\rho}^{v} T_{\theta}^{\theta \mu}\right) \\
T_{\mu \nu}^{\lambda}=\Gamma_{\mu \nu}^{\rho}-\Gamma_{\nu \mu}^{\rho}=h_{i}^{\lambda}\left(\partial_{\mu} h_{\nu}^{i}-\partial_{\nu} h_{\mu}^{i}\right)
\end{gathered}
$$

where $\Gamma_{\nu \mu}^{\rho}$ is the Weitzenböck connection.

A discretionary option in choosing the vierbein field related to the metric tensor $g_{\mu v}$ by the following relation

$$
g_{\mu v}=\eta_{i j} h_{\mu}^{i} h_{v}^{i} \quad i, j, \ldots=0,1,2,3 ; \mu, v, \ldots=0,1,2,3 .
$$

where the field $h_{i}\left(x^{\mu}\right)$ at every point $x^{\mu}$ of manifold are orthonormal basis for the space- time and $e_{i} e_{j}=n_{i j}$, where $n_{i j}=\operatorname{diag}(1,-1,-1,-1)$ is the Minkowski metric.

Here, $h_{\mu}^{i}$ satisfies the properties

$$
h_{\mu}^{i} h_{j}^{\mu}=\delta_{j}^{i}, \quad h_{\mu}^{i} h_{i}^{\mu}=\delta_{\mu}^{v}
$$

The contorsion tensor $K_{\rho}^{\mu \nu}$ has the following form

$$
K_{\rho}^{\mu \nu}=-\frac{1}{2}\left(T_{\rho}^{\mu \nu}-T_{\rho}^{\nu \mu}-T_{\rho}^{\mu \nu}\right)
$$

where contorsion tensor is the connection of difference between Weitzenböck and Levi-Civita.

Now, we present the field equations of $F(T)$ theory of gravity for the action (2) with respect to tetrad field

$$
\left[e^{-1} \partial_{\mu}\left(e S_{i}^{\mu \nu}\right)-h_{i}^{\lambda} T_{\mu \lambda}^{\rho} S_{\rho}^{\nu \mu}\right] F_{T}+S_{i}^{\mu \nu} \partial_{\mu}(T) F_{T T}+\frac{1}{4} h_{i}^{v} F=\frac{1}{2} k^{2} h_{i}^{\rho} T_{\rho}^{v}
$$

where $F(T)$ is the general differentiable function of torsion, $k^{2}=8 \pi G, F_{T}=\frac{d F}{d T} \quad, \quad S_{i}{ }^{\mu}=h_{i}^{\rho} S_{\rho}{ }^{\mu \nu}$.

The energy momentum tensor has the components

$$
T_{\rho}^{v}=\operatorname{diag}\left(\rho_{m},-p_{m},-p_{m},-p_{m}\right)
$$

where $\rho_{m}$ and $p_{m}$ are energy density and pressure of matter inside the universe respectively.

\section{The Field Equations}

Now we assume the line element for homogenous and anisotropic LRS Bianchi type-I space-time having one transverse direction $x$ and two equal longitudinal directions $y$ and $z$ which are responsible for anisotropic behavior [47] 


$$
d s^{2}=d t^{2}-A^{2}(t) d x^{2}-B^{2}(t)\left(d y^{2}+d z^{2}\right)
$$

where $A(t)$ and $B(t)$ are the cosmic scale factors.

We obtain the vierbein components by using equation (6) and equation (11) as

$$
h_{\mu}^{i}=\operatorname{diag}(1, A, B, B), \quad h_{i}^{\mu}=\operatorname{diag}\left(1, A^{-1}, B^{-1}, B^{-1}\right)
$$

By using equations (4) and (5) in equation (3), the torsion tensor for LRS Bianchi type-I has the form

$$
T=-2\left(2 \frac{\dot{A} \dot{B}}{A B}+\frac{\dot{B}^{2}}{B^{2}}\right)
$$

The above field equations (9) of $F(T)$ theory of gravity, using equations (10)-(13) reduce to the following set of equations for $i=0=v$ and $i=1=v$,

$$
\begin{gathered}
F-4\left(2 \frac{\dot{A} \dot{B}}{A B}+\frac{\dot{B}^{2}}{B^{2}}\right) F_{T}=2 k^{2} \rho_{m} \\
4\left(\frac{\dot{A} \dot{B}}{A B}+\frac{\dot{B}^{2}}{B^{2}}+\frac{\ddot{B}}{B}\right) F_{T}-16 \frac{\dot{B}}{B}\left[\frac{\dot{B}}{B}\left(\frac{\ddot{A}}{A}-\frac{\dot{A}^{2}}{A^{2}}\right)+\left(\frac{\ddot{B}}{B}-\frac{\dot{B}^{2}}{B^{2}}\right)\left(\frac{\dot{B}}{B}+\frac{\dot{A}}{A}\right)\right] F_{T T}-F=2 k^{2} p_{m}
\end{gathered}
$$

The conservation equation of energy momentum tensor turns out to be

$$
\dot{\rho}_{m}+\left(\frac{\dot{A}}{A}+2 \frac{\dot{B}}{B}\right)\left(\rho_{m}+p_{m}\right)=0
$$

The directional Hubble parameter $H_{i}$ in the direction of $x, y$ and $z$ axes respectively are defined as

$$
H_{1}=\frac{\dot{A}}{A}, H_{2}=H_{3}=\frac{\dot{B}}{B}
$$

We have defined the average scale factor $R$, the Hubble parameter $H$ and the anisotropy parameter $\Delta$ for LRS Bianchi type-I by

$$
\begin{gathered}
R=\left(A B^{2}\right)^{1 / 3} \\
H=\frac{1}{3}\left(\frac{\dot{A}}{A}+2 \frac{\dot{B}}{B}\right) \\
\Delta=\frac{1}{3} \sum_{i=1}^{3}\left(\frac{H_{i}-H}{H}\right)^{2}
\end{gathered}
$$

For $\Delta=0$, it is observed that isotropic behavior of the universe is obtained, which depends on the values of unknown scale factors and parameters that are involved in the model [47-49].

Using equations (13) and (19), we get

which is equal to

$$
T=-9 H^{2}+J \text { with } J=\frac{\dot{A}^{2}}{A^{2}}+2 \frac{\dot{B}^{2}}{B^{2}}
$$

$$
H=\frac{1}{3} \sqrt{J-T}
$$

Now using $F(T)=T, F_{T}=1$ in equations (14) and (15), we get

$$
\begin{gathered}
\rho_{m}+\rho_{T}=\frac{1}{2 k^{2}}\left[-4\left(2 \frac{\dot{A} \dot{B}}{A B}+\frac{\dot{B}^{2}}{B^{2}}\right)+T\right] \\
p_{m}+p_{T}=\frac{1}{2 k^{2}}\left[4\left(\frac{\dot{A} \dot{B}}{A B}+\frac{\dot{B}^{2}}{B^{2}}+\frac{\ddot{B}}{B}\right)-T\right]
\end{gathered}
$$

where $\rho_{T}$ and $p_{T}$ are the torsion contribution to the energy density and pressure given by 


$$
\begin{gathered}
\rho_{T}=\frac{1}{2 k^{2}}\left[-4\left(2 \frac{\dot{A} \dot{B}}{A B}+\frac{\dot{B}^{2}}{B^{2}}\right)\left(1-F_{T}\right)+T-F\right] \\
p_{T}=\frac{1}{2 k^{2}}\left[4\left(\frac{\dot{A} \dot{B}}{A B}+\frac{\dot{B}^{2}}{B^{2}}+\frac{\ddot{B}}{B}\right)\left(1-F_{T}\right)+16 \frac{\dot{B}}{B}\left\{\frac{\dot{B}}{B}\left(\frac{\ddot{A}}{A}-\frac{\dot{A}^{2}}{A^{2}}\right)+\left(\frac{\ddot{B}}{B}-\frac{\dot{B}^{2}}{B^{2}}\right)\left(\frac{\dot{B}}{B}+\frac{\dot{A}}{A}\right)\right\}\right.
\end{gathered}
$$

We get the solution from the homogeneous part of equation (14) i.e. $\rho=0$ as

$$
F(T)=\frac{c_{0}}{\sqrt{T}}
$$

where $c_{0}$ is the constant of integration.

From this solution, equation (15) takes the form

$$
p_{m}=\frac{1}{2 k^{2}}\left[\frac{6 \chi \dot{T}}{T^{2}}-\frac{3 \dot{H}+J+\tau}{T}-\frac{1}{2}\right] \frac{c_{0}}{\sqrt{T}}
$$

where $\chi=\frac{\dot{B}}{B}, \tau=\frac{\dot{B}^{2}}{B^{2}}$.

\section{Construction of Some $F(T)$ Models Using Continuity Equation}

In this section by using continuity equation (16), we have constructed the $F(T)$ models for different cases of perfect fluid and discussed the values of EoS parameter for non-relativistic matter, radiation and D.E.

Here for LRS Bianchi type-I universe, we use equation [50]

$$
\frac{1}{9}\left(\frac{\dot{A}}{A}+2 \frac{\dot{B}}{B}\right)^{2}=H_{0}^{2}+\frac{k^{2} \rho_{0}}{3 A B^{2}}
$$

It implies

$$
\left(A B^{2}\right)^{-1}=\frac{3}{k^{2} \rho_{0}}\left(H^{2}-H_{0}^{2}\right)
$$

where $\rho_{0}$ is the constant of integration and $H_{0}$ is the Hubble constant.

Equation (16) can be written in terms of EoS parameter

$$
\frac{\dot{\rho}_{m}}{\rho_{m}}+3 H(1+\omega)=0
$$

Now we construct the $F(T)$ models for the different cases of fluids and their combination which are for relativistic matter, non-relativistic matter and DE era [51].

Case 1: For non-relativistic matter i.e. $\omega=0$. It represents cold dark matter (CDM) and baryons.

In equation (31), we put $\omega=0$ and from equation (30), we get

$$
\rho_{m}=\rho_{c}\left(A B^{2}\right)^{-1}=\frac{3 \rho_{c}}{k^{2} \rho_{0}}\left(H^{2}-H_{0}^{2}\right)
$$

since $\rho_{c}$ is a constant of integration.

Also, equation (32) in the form of torsion scalar is

$$
\rho_{m}=\frac{\rho_{c}}{3 k^{2} \rho_{0}}\left(J-9 H_{0}^{2}-T\right)
$$

By substituting the values of $\rho_{m}$ in equation (14) we get

$$
2 T F_{T}+F=\frac{2 \rho_{c}}{3 \rho_{0}}\left(J-9 H_{0}^{2}-T\right)
$$

Solution of the above equation is 


$$
F(T)=\frac{2 \rho_{c}}{3 \rho_{0}}\left(J-9 H_{0}^{2}-T\right)
$$

Equation (35) gives a unique solution if the unknown scale factor $J$ is known.

So, here we get the model in terms of torsion and Hubble constant.

Case2: For relativistic matter i.e. $\omega=\frac{1}{3}$ which represents photons and massless neutrinos. It indicates the radiation dominated era of the universe.

Putting $\omega=\frac{1}{3}$ in equation (31) and by using equation (21) and equation (30), we get

$$
\rho_{m}=\frac{\rho_{r}}{3^{4 / 3} k^{8 / 3} \rho_{0}^{4 / 3}}\left(J-9 H_{0}^{2}-T\right)^{4 / 3}
$$

where $\rho_{r}$ is another constant of integration.

Substituting the values of $\rho_{m}$ in equation (14), it implies

$$
2 T F_{T}+F=\frac{2 \rho_{r}}{3^{4 / 3} k^{2 / 3} \rho_{0}^{4 / 3}}\left(J-9 H_{0}^{2}-T\right)^{4 / 3}
$$

Equation (37) has the solution

$$
F(T)=\frac{2 \rho_{r}}{3^{4 / 3} k^{2 / 3} \rho_{0}^{4 / 3}}\left(J-9 H_{0}^{2}-T\right)^{4 / 3}
$$

Here also the solution depends upon the values of $J$, torsion scalar $T$ and Hubble constant.

Case 3: For DE era i.e. $\omega=-1$. This represents the major component of the universe i.e. the DE component which has a large negative pressure.

Now by using $\omega=-1$ in equation (31), we get

$$
\rho_{m}=\rho_{d}
$$

where $\rho_{d}$ is the constant of integration. From equation (39), equation (14) implies that

$$
2 T F_{T}+F=2 k^{2} \rho_{d}
$$

Solution of the above equation is

$$
F(T)=2 k^{2} \rho_{d}
$$

This solution gives the constant model which is consistent with cosmological constant.

Case 4: Combination of Dust fluid and radiation i.e. $\omega=0$ and $\omega=\frac{1}{3}$. Here we take the combination of two different fluids, the dust fluid and the radiations.

By adding equation (33) and equation (36) we get

$$
\rho_{m}=\frac{1}{3 k^{2} \rho_{0}}\left(J-9 H_{0}^{2}-T\right)\left[\rho_{c}+\frac{\rho_{r}}{3^{1 / 3} k^{2 / 3} \rho_{0}^{1 / 3}}\left(J-9 H_{0}^{2}-T\right)^{1 / 3}\right]
$$

Put the value of $\rho_{m}$ in equation (14), it follows that

$$
2 T F_{T}+F=\frac{2}{3 \rho_{0}}\left(J-9 H_{0}^{2}-T\right)\left[\rho_{c}+\frac{\rho_{r}}{3^{1 / 3} k^{2 / 3} \rho_{0}^{1 / 3}}\left(J-9 H_{0}^{2}-T\right)^{1 / 3}\right]
$$

We obtain

$$
F(T)=\frac{2}{3 \rho_{0}}\left(J-9 H_{0}^{2}-T\right)\left[\rho_{c}+\frac{\rho_{r}}{3^{1 / 3} k^{2 / 3} \rho_{0}^{1 / 3}}\left(J-9 H_{0}^{2}-T\right)^{1 / 3}\right]
$$

Case 5: Combination of Dust fluid and DE i.e. $\omega=0$ and $\omega=-1$, which gives 


$$
\rho_{m}=\frac{\rho_{c}}{3 k^{2} \rho_{0}}\left(J-9 H_{0}^{2}-T\right)+\rho_{d}
$$

By using equation (45), equation (14) takes the form

$$
2 T F_{T}+F=\frac{2 \rho_{c}}{3 \rho_{0}}\left(J-9 H_{0}^{2}-T\right)+2 k^{2} \rho_{d}
$$

This gives

$$
F(T)=\frac{2 \rho_{c}}{3 \rho_{0}}\left(J-9 H_{0}^{2}-T\right)+2 k^{2} \rho_{d}
$$

Case 6: Combination of DE and Radiation i.e. $\omega=-1$ and $\omega=\frac{1}{3}$.

In this section we consider the combination of EoS parameter for DE and radiation dominated era which yields

$$
\rho_{m}=\rho_{d}+\frac{\rho_{r}}{3^{4 / 3} k^{8 / 3} \rho_{0}^{4 / 3}}\left(J-9 H_{0}^{2}-T\right)^{4 / 3}
$$

Using equation (48) in equation (14), we have

$$
2 T F_{T}+F=2 k^{2} \rho_{d}+\frac{2 \rho_{r}}{3^{4 / 3} k^{2 / 3} \rho_{0}^{4 / 3}}\left(J-9 H_{0}^{2}-T\right)^{4 / 3}
$$

It implies the following solution

$$
F(T)=2 k^{2} \rho_{d}+\frac{2 \rho_{r}}{3^{4 / 3} k^{2 / 3} \rho_{0}^{4 / 3}}\left(J-9 H_{0}^{2}-T\right)^{4 / 3}
$$

\section{Conclusion}

By using continuity equation, some $F(T)$ gravity LRS Bianchi type-I models have been constructed. These $F(T)$ gravity models represent three different phases such as matter, radiation and DE respectively corresponding to $\omega=0, \omega=1 / 3$ and $\omega=-1$. Matter dominated era explains expansion of the universe filled with non-interacting dust particles while radiation dominated era represents early universe filled with radiation. The DE era corresponds to the universe dominated by a strong negative pressure causing late time acceleration. An attempt has been made for the revival of the form used by Sharif \& Rani [1]. Our results are analogous to the results obtained by Sharif and Rani [1].

\section{References}

1. M. Sharif, and S. Rani, "F(T) Models within Bianchi type-I Universe," Modern Physics Letters A, vol. 26, no. 22, pp. 1657-1671, 2011.

2. S. Perlmutter, S. Gabi, G. Goldhaber, A. Goobar, D. E. Groom, I. M. Hook, A. G. Kim, M. Y. Kim, J. C. Lee, R. Pain and C. R. Pennypacker, "Measurements Based in part on data from the Isaac Newton Group Telescopes, KPNO and CTIO Observatories run by AURA, Mount Stromlo \& Siding Spring Observatory, Nordic Optical Telescope, and the WM Keck Observatory of the Cosmological Parameters $\Omega$ and $\Lambda$ from the First Seven Supernovae at $\mathrm{z} \geqslant 0.3$," The Astrophysical Journal, vol. 483, no. 2, pp. 565, 1997.

3. S. Perlmutter, G. Aldering, M. Della Valle, S. Deustua, R. S. Ellis, S. Fabbro, A. Fruchter, G. Goldhaber, D. E. Groom, I. M. Hook and A. G. Kim, "Discovery of a supernova explosion at half the age of the Universe," Nature, vol. 391, no. 6662), pp. 51-54, 1998.

4. S. Perlmutter, G. Aldering, G. Goldhaber, R. A. Knop, P. Nugent, P. G. Castro, S. Deustua, S. Fabbro, A. Goobar, D. E. Groom and I. M. Hook, "Measurements of $\Omega$ and $\Lambda$ from 42 high-redshift supernovae,' The Astrophysical Journal, vol. 517, no.2, pp. 565, 1999. 
5. A. G. Riess, A. V. Filippenko, P. Challis, A. Clocchiatti, A. Diercks, P. M. Garnavich, R. L. Gilliland, C. J. Hogan, S. Jha, R. P. Kirshner and B.R.U.N.O. Leibundgut, "Observational evidence from supernovae for an accelerating universe and a cosmological constant," The Astronomical Journal, vol. 116, no. 3, pp. 1009, 1998.

6. A.G. Riess, L.G. Strolger, J. Tonry, S. Casertano, H.C. Ferguson, B. Mobasher, , P. Challis, A.V. Filippenko, S. Jha, W. Li and R. Chornock, "Type Ia Supernova Discoveries at z> 1 from the Hubble Space Telescope: Evidence for Past Deceleration and Constraints on Dark Energy EvolutionBased on observations with the NASA/ESA Hubble Space Telescope, obtained at the Space Telescope Science Institute, which is operated by AURA, Inc., under NASA contract NAS5-26555," The Astrophysical Journal, vol. 607, pn. 2, pp. 665, 2004.

7. C. L. Bennett, M. Halpern, G. Hinshaw, N. Jarosik, A. Kogut, M. Limon, S. S. Meyer, L. Page, D. N. Spergel, G. S. Tucker and E. Wollack, "First-Year Wilkinson Microwave Anisotropy Probe (WMAP) WMAP is the result of a partnership between Princeton University and the NASA Goddard Space Flight Center. Scientific guidance is provided by the WMAP Science Team. Observations: Preliminary Maps and Basic Results," The Astrophysical Journal Supplement Series, vol. 148, no. 1, pp. 1, 2003.

8. E. Komatsu, A. Kogut, M. R. Nolta, C. L. Bennett, M. Halpern, G. Hinshaw, N. Jarosik, M. Limon, S. S. Meyer, L. Page and D. N. Spergel, "First-Year Wilkinson Microwave Anisotropy Probe (WMAP) WMAP is the result of a partnership between Princeton University and the NASA Goddard Space Flight Center. Scientific guidance is provided by the WMAP Science Team. Observations: Tests of Gaussianity," The Astrophysical Journal Supplement Series, vol. 148, no. 1, pp. 119, 2003.

9. D. N. Spergel, R. Bean, O. Doré, M.R. Nolta, C.L. Bennett, J. Dunkley, G. Hinshaw, N. Jarosik, E. Komatsu, L. Page and H.V. Peiris, "Three-year Wilkinson Microwave Anisotropy Probe (WMAP) observations: implications for cosmology," The Astrophysical Journal Supplement Series, vol.170, no.2,pp. 377, 2007.

10. E. Hawkins, S. Maddox, S. Cole, O. Lahav, D. S. Madgwick, P. Norberg, J. A. Peacock, I. K. Baldry, C. M. Baugh, J. Bland-Hawthorn and T. Bridges, "The 2dF Galaxy Redshift Survey: correlation functions, peculiar velocities and the matter density of the Universe," Monthly Notices of the Royal Astronomical Society, vol. 346,no.1, pp.78-96.2003.

11. M. Tegmark, M. A. Strauss, M. R. Blanton, K. Abazajian, S. Dodelson, H. Sandvik, X. Wang, D.H., Weinberg, I. Zehavi, N.A. Bahcall and F. Hoyle, "Cosmological parameters from SDSS and WMAP," Physical Review D, vol. 69, no. 10, pp. 103501, 2004.

12. S. Cole, W. J. Percival, J. A. Peacock, P. Norberg, C. M. Baugh, C. S. Frenk, I. Baldry, J. Bland-Hawthorn, T. Bridges, R. Cannon and M. Colless, "The 2dF Galaxy Redshift Survey: power-spectrum analysis of the final data set and cosmological implication," Monthly Notices of the Royal Astronomical Society, vol. 362, no. 2, pp. 505-534, 2005.

13. P. A. R. Ade, N. Aghanim, C. Armitage-Caplan, M. Arnaud, M. Ashdown, F. Atrio-Barandela, J. Aumont, C. Baccigalupi, A.J. Banday, R.B. Barreiro and J.G. Bartlett, "Planck 2013 results. XVI. Cosmological parameters," Astronomy \& Astrophysics, vol. 571, pp. A16, 2014.

14. L. Yang, C.C. Lee, L. W. Luo and C. Q. Geng, "Observational constraints on exponential gravity," Physical Review D, vol. 82, no. 10, pp. 103515, 2010.

15. S. I. Nojiri, and S. D. Odintsov, "EConf: Electron. Conf," Proc. Arch C, vol. 602061, pp. 06, 2006.

16. S. I. Nojiri, and S.D. Odintsov, "Introduction to modified gravity and gravitational alternative for dark energy," International Journal of Geometric Methods in Modern Physics, vol.4, no. 01, pp. 115-145, 2007.

17. S. I. Nojiri and S. D. Odintsov, "Unified cosmic history in modified gravity: from $F(R)$ theory to Lorentz noninvariant models," Physics Reports, vol. 505, no. 2, pp. 59-144, 2011.

18. T. P. Sotiriou And V. Faraoni, " $f(R)$ theories of gravity," Reviews of Modern Physics, vol. 82, no. 1, pp. 451, 2010.

19. A. De Felice and S. Tsujikawa, " $f(R)$ theories," Living Rev. Rel, vol. 13, no. 3, pp.1002-4928, 2010.

20. T. Clifton, P. G. Ferreira, A. Padilla and C. Skordis, "Modified gravity and cosmology," arXiv preprint, arXiv:1106.2476, 2011.

21. T. Harko, F. S. Lobo, S. I. Nojiri and S. D. Odintsov, " $f(R, T)$ gravity," Physical Review D, vol. 84, no. 2, pp. 024020, 2011

22. R. Ferraro and F. Fiorini, "Modified teleparallel gravity: inflation without an inflaton," Physical Review D, 75(8), p.084031, 2007. 
23. R. Ferraro and F. Fiorini, "Born-Infeld gravity in Weitzenböck spacetime," Physical Review D, vol. 78, no. 12, pp. 124019, 2008.

24. S. H. Chen, J. B. Dent, S. Dutta and E. N. Saridakis, "Cosmological perturbations in $f(T)$ gravity," Physical Review D, vol. 83, no. 2, pp. 023508, 2011.

25. B. Li, T. P. Sotiriou and J. D. Barrow, " $f(T)$ gravity and local Lorentz invariance," Physical Review D, vol. 83, no. 6, pp. 064035, 2011

26. H. R. Kausar, I. Noureen and M. U. Shahzad, "Dynamical analysis of charged anisotropic spherical star in $f(R)$ gravity," The European Physical Journal Plus, vol. 130, no. 10, pp. 1-10, 2015.

27. S. D. Odintsov and V. K. Oikonomou, "Bouncing cosmology with future singularity from modified gravity," Physical Review D, vol. 92, no. 2, pp. 024016, 2015.

28. S. D. Odintsov, V. K. Oikonomou and E. N. Saridakis, "Superbounce and loop quantum ekpyrotic cosmologies from modified gravity: $F(R), F(G)$ and $F(T)$ theories," Annals of Physics, vol. 363, pp. 141-163, 2015.

29. G. Abbas, D. Momeni, M. A. Ali, R. Myrzakulov and S. Qaisar, "Anisotropic compact stars in $f(G)$ gravity." Astrophysics and Space Science, vol. 357, no. 2, pp. 1-11, 2015.

30. S. B. Nassur, A. V. Kpadonou, M. E. Rodrigues, M. J. S. Houndjo and J. Tossa, "Realistic $f(T)$ model describing the de Sitter epoch of the dark energy dominated universe," Canadian Journal of Physics, vol. 93, no. 10, pp. 1050-1056, 2015.

31. J. J. Geng, R. Y. Guo, D. Z. He, J. F. Zhang and X. Zhang, "Redshift drift constraints on $f(T)$ gravity," Frontiers of Physics, vol. 10, no. 5, pp. 1-6, 2015.

32. A. Das, F. Rahaman, B. K. Guha and S. Ray, "Relativistic compact stars in $f(T)$ gravity admitting conformal motion," Astrophysics and Space Science, vol. 358, no. 2, pp. 1-8, 2015.

33. G. L. Nashed, G.L., "FRW in quadratic form of $f(T)$ gravitational theories," General Relativity and Gravitation, vol. 47, no. 7, pp. 1-14, 2015.

34. G. Hinshaw, "First year Wilkinson microwave anisotropy probe (WMAP) observations: angular power spectrum," Astrophys. J. Suppl, vol. 148, pp. 135, 2003.

35. G. Hinshaw, M. R. Nolta, C. L. Bennett, R. Bean, O. Dore, M. R. Greason, M. Halpern, R. S. Hill, N. Jarosik, A. Kogut and E. Komatsu, "Three-Year Wilkinson Microwave Anisotropy Probe (WMAPWMAP is the result of a partnership between Princeton University and the NASA Goddard Space Flight Center. Scientific guidance is provided by the WMAP Science Team.) Observations: Temperature Analysis," The Astrophysical Journal Supplement Series, vol. 170, no. 2, pp. 288, 2007.

36. G. Hinshaw, J. L. Weiland, R. S. Hill, N. Odegard, D. Larson, C. L. Bennett, J. Dunkley, B. Gold, M. R. Greason, N. Jarosik, and E. Komatsu, "FIVE-YEAR WILKINSON MICROWAVE ANISOTROPY PROBE WMAP is the result of a partnership between Princeton University and NASA's Goddard Space Flight Center. Scientific guidance is provided by the WMAP Science Team. OBSERVATIONS: DATA PROCESSING, SKY MAPS, AND BASIC RESULTS," The Astrophysical Journal Supplement Series, vol. 180, no. 2, pp. $225,2009$.

37. S. Kumar and C. P. Singh, "Anisotropic Bianchi type-I models with constant deceleration parameter in general relativity," Astrophysics and Space Science, vol. 312, no. 1-2, pp. 57-62, 2007.

38. S. Kumar and C. P. Singh, "Exact bianchi type-I cosmological models in a scalar-tensor theory," International Journal of Theoretical Physics, vol. 47, no. 6, pp. 1722-1730, 2008.

39. M. Sharif and S. Waheed, "Anisotropic universe models in Brans-Dicke theory," The European Physical Journal $\boldsymbol{C}$, vol. 72, no. 2, pp. 1-12, 2012.

40. R. Bali and P. Kumawat, "Bulk viscous LRS Bianchi type V tilted stiff fluid cosmological model in general relativity," Physics Letters $\boldsymbol{B}$, vol. 665, no. 5, pp. 332-337, 2008.

41. H. Amirhashchi, "LRS Bianchi type II stiff fluid cosmological model with decaying vacuum energy density $\Lambda$ in general relativity," Physics Letters B, vol. 697, no. 5, pp. 429-433, 2011.

42. A. K. Yadav and B. Saha, "LRS Bianchi-I anisotropic cosmological model with dominance of dark energy," Astrophysics and Space Science, vol. 337, no. 2, pp. 759-765, 2012.

43. K. S. Adhav, "LRS Bianchi type-I cosmological model with linearly varying deceleration parameter," The European Physical Journal Plus, vol. 126, no. 12, pp. 1-5, 2011.

44. K. S. Adhav, "LRS Bianchi Type-I universe with anisotropic dark energy in lyra geometry," International Journal of Astronomy and Astrophysics, vol. 1, no. 04, pp. 204, 2011. 
45. K. S. Adhav, "LRS Bianchi type-I cosmological model in $f(R, T)$ theory of gravity," Astrophysics and Space Science, vol. 339, no. 2, pp. 365-369, 2012.

46. K. S. Adhav, A. S. Bansod, M. S. Desale, R. B. Raut, "LRS Bianchi type-I models with constant deceleration parameter in creation field cosmology," Astrophysics and Space Science, vol. 331, no. 2, pp. 689-695, 2011.

47. M. Sharif and M. Zubair, "Dynamics of Bianchi I universe with magnetized anisotropic Dark Energy," Astrophysics and Space Science, vol. 330, no. 2, pp. 399-405, 2010.

48. M. Sharif and H. R. Kausar, "Anisotropic fluid and Bianchi type III model in $f(R)$ gravity," Physics Letters $B$, vol. 697, no. 1, pp. 1-6, 2011.

49. R. K. Tiwari, "Bianchi type-I cosmological models with perfect fluid in general relativity," Research in Astronomy and Astrophysics, vol. 10, no. 4, pp. 291, 2010.

50. E. Elizalde, R. Myrzakulov, V. V. Obukhov and D. Sáez-Gómez, " $\Lambda$ CDM epoch reconstruction from $F(R, G)$ and modified Gauss-Bonnet gravities," Classical and Quantum Gravity, vol. 27, no. 9, pp. 095007, 2010.

51. R. Bean, "TASI lectures on cosmic acceleration," arXiv preprint, arXiv:1003.4468, 2010. 